Comm. Math. Phys., 207, N1, (1999), 231-247.

\title{
AN INVERSE SCATTERING PROBLEM WITH PART OF THE FIXED-ENERGY PHASE SHIFTS
}

\author{
A.G. RAMM \\ Mathematics Department, Kansas State University, Manhattan, KS 66506, USA \\ email: ramm@math.ksu.edu
}

\begin{abstract}
Assume that $q(r)$ is a real-valued, compactly supported potential, $q(r)=0$ for $r:=|x|>a$, $q(r) \in L^{2}\left(B_{a}\right), n=3, B_{a}:=\left\{x: x \in \mathbb{R}^{n},|x|<a\right\}$. Let $\mathcal{L}$ be an arbitrary fixed subset of non-negative integers such that $\sum_{\ell \neq 0, \ell \in \mathcal{L}} \frac{1}{\ell}=\infty$, and $\left\{\delta_{\ell}\right\}$ be fixed-energy phase shifts corresponding to $q(r)$. The main result is:
\end{abstract}

Theorem. The data $\left\{\delta_{\ell}\right\}_{\forall \ell \in \mathcal{L}}$ determine $q(r)$ uniquely.

\section{INTRODUCTION}

In this paper a uniqueness theorem is established for inverse scattering with fixed energy data. This theorem says that a real-valued compactly supported spherically symmetric potential $q(r)$ is uniquely determined by the subset of the phase shifts $\delta_{\ell}$ at an arbitrary fixed positive energy. The subset $\left\{\delta_{\ell}\right\}_{\ell \in \mathcal{L}}$ is defined by an arbitrary subset $\mathcal{L}$ of integers $\{0,1,2, \ldots\}$, with the property

$$
\sum_{\ell \in \mathcal{L}, \ell \neq 0} \frac{1}{\ell}=\infty
$$

No results of this type have been known or conjectured earlier.

Condition (1.1) appeared in Müntz's theorem: it is a necessary and sufficient condition for the completeness of the set $\left\{x^{\ell}\right\}$ in $L^{1}(0, a)$, for an arbitrary fixed $a>0$.

Such a result gives much deeper understanding of the quantum-mechanical inverse scattering problem with fixed-energy data. It may also be of some practical significance because in some physical experiments the phase shifts can be measured not for all $\ell$ and it is important to know what part of the fixed-energy phase shifts is still sufficient for the unique identification of the potential.

We now describe the basic ideas of the proof. Our proof is based on two fundamental results.

The first result is the uniqueness theorem from [R1] which says that the fixed-energy scattering data $A\left(\alpha^{\prime}, \alpha\right), \forall \alpha^{\prime}, \alpha \in S^{2}$, determine $q(x) \in Q_{a}$ uniquely. Here $A\left(\alpha^{\prime}, \alpha\right)$ is the scattering amplitude corresponding to the potential $q(x) \in Q_{a}:=\left\{q: q=\bar{q}, q(x)=0\right.$ for $\left.|x|>a, q(x) \in L^{2}\left(B_{a}\right)\right\}$, where $B_{a}:=\left\{x:|x| \leq a, x \in \mathbb{R}^{3}\right\}$ and the bar stands for complex conjugate. Under this assumption one can check, using Hölder's inequality, that $\int_{0}^{a} r|q(r)| d r<\infty$. The proof of the above uniqueness theorem is valid in $\mathbb{R}^{n}, n \geq 3$, and is not valid for $n=2$ (see [R2] for an explanation). An algorithm for inversion of noisy fixed-energy scattering data is developed in [R5] (see also [R2]), where a stability estimate for this algorithm is obtained. In [A] a discussion of the Newton-Sabatier method for inversion of fixed-energy phase shifts is given and an example is constructed of two quite different compactly supported piecewiseconstant potentials which produce practically the same ( within the accuracy $10^{-5}$ ) fixed-energy phase shifts for all values of the angular momenta. This example illustrates the stability estimate from [R5] and shows that the above estimate is sharp.

Actually, property $C$ for a pair of Schrödinger operators is used for a proof of the uniqueness theorem for inverse scattering problem with fixed-energy data. This theorem follows from property $C$. The notion

1991 Mathematics Subject Classification. 35R30, 34B25, 34A55, 81F05,81F15.

Key words and phrases. : fixed-energy phase shifts, inverse scattering.

Typeset by $\mathcal{A}_{\mathcal{M}} \mathcal{S}-\mathrm{T}_{\mathrm{EX}}$ 
of the property $C$ has been introduced and applied to many inverse problems in a series of papers by the author (see [R2] and references therein, [R3]-[R4]).

Let us formulate this notion for a pair $\left\{L_{1}, L_{2}\right\}$ of the Schrödinger operators $L_{j}=-\nabla^{2}+q_{j}-k^{2}, j=$ $1,2, q_{j} \in Q_{a}, k \geq 0$ is a constant, $k^{2}$ is the energy of the particle.

Let $D \subset \mathbb{R}^{n}$ be a bounded domain and

$$
N_{j}:=N_{D}\left(L_{j}\right):=\left\{w: L_{j} w=0 \text { in } D, w \in H^{2}(D)\right\} .
$$

Let $f \in L^{2}(D)$.

Definition 1. If

$$
\left\{\int_{D} f(x) w_{1}(x) w_{2}(x) d x=0 \quad \forall w_{j} \in N_{j}\right\} \Rightarrow f=0,
$$

then we say that the pair $\left\{L_{1}, L_{2}\right\}$ has property $C$.

Let $n=3$ and $S^{2}$ be the unit sphere in $\mathbb{R}^{3}$.

Define the scattering solution corresponding to the operator $L=-\nabla^{2}+q-k^{2}$, and a fixed $k>0$, which we take, without loss of generality, to be $k=1$ in this paper, as the solution to the equation:

$$
\begin{gathered}
{\left[\nabla^{2}+1-q(x)\right] \psi(x, \alpha)=0 \text { in } \mathbb{R}^{3}} \\
\psi(x, \alpha)=\exp (i x \cdot \alpha)+A\left(\alpha^{\prime}, \alpha\right) \frac{e^{i r}}{r}+o\left(\frac{1}{r}\right), \quad r:=|x| \rightarrow \infty, \quad \alpha^{\prime}:=\frac{x}{r} .
\end{gathered}
$$

The unit vector $\alpha$ is given, the coefficient $A\left(\alpha^{\prime}, \alpha\right)$ is called the scattering amplitude. It is well known that $q(x) \in Q_{a}$ determines $A\left(\alpha^{\prime}, \alpha\right)$ uniquely.

It is proved in $[\mathrm{R} 2]$ that

$$
\left\{\int_{D} f(x) \psi_{1}(x, \alpha) \overline{\psi_{2}(x, \beta)} d x=0 \quad \forall \alpha, \beta \in S^{2}\right\} \Rightarrow f=0,
$$

where $\psi_{j}(x, \alpha), j=1,2$, is the scattering solution corresponding to the operator $L=-\nabla^{2}+q_{j}-k^{2}, j=$ 1,2 , and a fixed $k=1$.

The inverse scattering problem (ISP) with fixed-energy data consists in finding $q(x) \in Q_{a}$ given $A\left(\alpha^{\prime}, \alpha\right) \quad \forall \alpha^{\prime}, \alpha \in S^{2}$.

Denote by $\tilde{S}^{2}$ an arbitrary small open subset of $S^{2}$.

Theorem 1.1. [R1]. The data $A\left(\alpha^{\prime}, \alpha\right) \quad \forall \alpha^{\prime} \in \tilde{S}_{1}^{2}, \forall \alpha \in \tilde{S}_{2}^{2}$, determine $q(x) \in Q_{a}$ uniquely.

Theorem 1.2. [R2]. If $q_{j} \in Q_{a}, j=1,2$, then (1.2) holds.

Theorem 1.2 implies that the pair $\left\{L_{1}, L_{2}\right\}$ of Schrödinger's operators with potentials $q_{j} \in Q_{a}$ does have property $C$.

The second result we will use is the uniqueness theorem for analytic functions.

Let us assume that $h(\ell)$ is a holomorphic function in $\Pi_{+}:=\{\ell: \operatorname{Re} \ell>0\}, \quad \ell=\sigma+i \tau, \quad \sigma \geq 0$, and $\tau$ are real numbers, $h(\ell) \in N$ (Nevanlinna class in $\left.\Pi_{+}\right)$, that is

$$
\sup _{0<r<1} \int_{-\pi}^{\pi} \ln ^{+}\left|h\left(\frac{1-r e^{i \varphi}}{1+r e^{i \varphi}}\right)\right| d \varphi<\infty
$$

where $\ln ^{+} x=\left\{\begin{aligned} \ln x & \text { if } \ln x>0, \\ 0 & \text { if } \ln x \leq 0 .\end{aligned}\right.$ 
Theorem 1.3. If $h(\ell) \in N$ then

$$
h(\ell)=0, \quad \forall \ell \in \mathcal{L},
$$

implies

$$
h(\ell) \equiv 0 \text { in } \Pi_{+},
$$

in particular

$$
h(\ell)=0 \quad \forall \ell=0,1,2, \ldots .
$$

Theorem 1.3 is a consequence of Theorem 1.4 which is formulated below. This Theorem in turn is an immediate corollary to Theorem 15.23 in [Ru, p.334].

Theorem 1.4. Assume that the following conditions hold:

i) $f(z)$ is holomorphic in the unit disc $D_{1}$,

ii) $f(z) \in N$ in $D_{1}$, that is

$$
\sup _{0<r<1} \int_{-\pi}^{\pi} \ln ^{+}\left|f\left(r e^{i \varphi}\right)\right| d \varphi<\infty
$$

iii) $f\left(z_{n}\right)=0, \quad n=1,2,3, \ldots$,

and

$$
\sum_{n=1}^{\infty}\left(1-\left|z_{n}\right|\right)=\infty
$$

Then

$$
f(z) \equiv 0 \text { in } D_{1} .
$$

Let us explain why Theorem 1.4 implies Theorem 1.3. Note that the function

$$
\ell=\frac{1-w}{1+w}
$$

maps conformally $D_{1}$ onto $\Pi_{+}$, while

$$
w=\frac{1-\ell}{1+\ell}
$$

is the inverse map $\Pi_{+} \rightarrow D_{1}$.

The function $h(\ell)=h\left(\frac{1-w}{1+w}\right):=f(w)$ is analytic in $D_{1}, \quad f(w) \in N$ in $D_{1}$, and $f\left(w_{\ell}\right)=0$, where $w_{\ell}=\frac{1-\ell}{1+\ell}$ and $h(\ell)=0$.

If $\ell \in \mathcal{L}$, then

$$
\sum_{\ell \in \mathcal{L}, \ell \neq 0}\left(1-\left|w_{\ell}\right|\right)=\sum_{\ell \in \mathcal{L}, \ell \neq 0}\left(1-\left|\frac{1-\ell}{1+\ell}\right|\right)=\sum_{\ell \in \mathcal{L}, \ell \neq 0} \frac{2}{\ell+1}=\infty
$$

because of the assumption (1.1). Thus, $f(w)=0$ in $D_{1}$ by Theorem 1.4, and therefore $h(\ell)=0$ in $\Pi_{+}$.

Thus, Theorem 1.3 follows from Theorem 1.4.

In order to describe the ideas of our proof, we need to state some known facts from the scattering theory. If $q(x)=q(r), r=|x|$, that is, the potential is spherically symmetric, and if $k=1$, then the scattering solution is:

$$
\psi(x, \alpha)=\sum_{\ell=0}^{\infty} 4 \pi i^{\ell} \frac{\psi_{\ell}(r)}{r} Y_{\ell}\left(x^{0}\right) \overline{Y_{\ell}(\alpha)}, \quad x^{0}:=\frac{x}{r},
$$

where $\psi_{\ell}(r)$ satisfies equations (1.17)-(1.19):

$$
\begin{gathered}
\psi_{\ell}^{\prime \prime}+\psi_{\ell}-\frac{\ell(\ell+1)}{r^{2}} \psi_{\ell}-q(r) \psi_{\ell}=0, \quad r>0 \\
\psi_{\ell}(r)=O\left(r^{\ell+1}\right) \text { as } r \rightarrow 0,
\end{gathered}
$$




$$
\psi_{\ell}(r)=e^{i \delta_{\ell}} \sin \left(r-\frac{\ell \pi}{2}+\delta_{\ell}\right)+o(1) \text { as } r \rightarrow \infty .
$$

Here $\delta_{\ell}$ is called the fixed-energy phase shift corresponding to the angular momentum $\ell$.

The functions $Y_{\ell}(\alpha)=Y_{\ell m}(\alpha), \quad-\ell \leq m \leq \ell$, in (1.12) are the spherical harmonics orthonormalized in $L^{2}\left(S^{2}\right)$. The summation in (1.16) and in (1.20) below includes the summation with respect to $m, \quad-\ell \leq$ $m \leq \ell$, and is not shown for brevity.

The corresponding scattering amplitude for the spherically symmetric potential is of the form:

$$
A\left(\alpha^{\prime}, \alpha\right)=A\left(\alpha^{\prime} \cdot \alpha\right)=\sum_{\ell=0}^{\infty} A_{\ell} Y_{\ell}\left(\alpha^{\prime}\right) \overline{Y_{\ell}(\alpha)}
$$

where

$$
A_{\ell}=2 \pi i\left(1-e^{2 i \delta_{\ell}}\right)=4 \pi e^{i \delta_{\ell}} \sin \delta_{\ell}
$$

Recall that we assume $k=1$ throughout.

Therefore, in the case of spherically symmetric potentials which we consider in this paper, there is a one-to-one correspondence between the scattering amplitude $A\left(\alpha^{\prime}, \alpha\right)$ and the set of numbers $\left\{A_{\ell}\right\}_{\ell=0,1,2, \ldots}$. The set $\left\{\delta_{\ell}\right\}_{\ell=0,1,2, \ldots,} \quad-\pi \leq \delta_{\ell}<\pi$, determines the set $\left\{A_{\ell}\right\}_{\ell=0,1,2, \ldots}$ uniquely, so there is a one-to-one correspondence between the scattering amplitude at a fixed energy and the set of all phase shifts with this energy.

Using (1.16) and the orthonormality of the spherical harmonics, one obtains:

$$
\left\{0=\int_{B_{a}} p(r) \psi_{1}(x, \alpha) \overline{\psi_{2}(x, \beta)} d x\right\} \Longleftrightarrow\left\{0=\sum_{\ell=0}^{\infty} \int_{0}^{a} p(r) \psi_{1 \ell}(r) \overline{\psi_{2 \ell}(r)} d r \overline{Y_{\ell}(\alpha)} Y_{\ell}(\beta), \quad \forall \alpha, \beta \in S^{2}\right\}
$$

where $p(r) \in Q_{a}$ is an arbitrary function.

Multiplying the second integral in (1.22) by $Y_{\ell}(\alpha)$, integrating over $S^{2}$ and using the orthonormality of the spherical harmonics, one gets that the first equality in (1.22) is equivalent to

$$
0=\int_{0}^{a} d r p(r) \psi_{1 \ell}(r) \overline{\psi_{2 \ell}(r)} \quad \forall \ell=0,1,2, \ldots
$$

The regular solution $\varphi_{\ell}(r)$ to equation (1.17) is defined uniquely by its behavior near the origin:

$$
\varphi_{\ell}(r)=\frac{r^{\ell+1}}{(2 \ell+1) ! !}+o\left(r^{\ell+1}\right), \quad r \rightarrow 0
$$

This solution is a real-valued function for $\ell=0,1,2, \ldots$ and $r>0$. Its behavior at infinity is known:

$$
\varphi_{\ell}=\left|F_{\ell}\right| \sin \left(r-\frac{\ell \pi}{2}+\delta_{\ell}\right)+o(1), \quad r \rightarrow+\infty,
$$

where $\delta_{\ell}$ is the same as in (1.19) and $\left|F_{\ell}\right|$ is a certain positive constant (the value of the Jost function $F_{\ell}(k)$ at $\left.k=1\right)$. Since $\psi_{\ell}(r)$ solves (1.17) and satisfies (1.18), it follows that

$$
\psi_{\ell}=c_{\ell} \varphi_{\ell}(r), \quad c_{\ell}=\text { const }
$$

Therefore condition (1.23) is equivalent to

$$
0=\int_{0}^{a} d r p(r) \varphi_{1 \ell}(r) \varphi_{2 \ell}(r):=h(\ell), \quad \ell=0,1,2, \ldots,
$$

where we have used the real-valuedness of $\varphi_{\ell}(r)$.

Let us now describe the idea of our proof. 
Step 1. Assuming that the data $\left\{\delta_{\ell}\right\}_{\ell \in \mathcal{L}}$ correspond to two different potentials $q_{1}(r)$ and $q_{2}(r), \quad q_{j}(r) \in$ $Q_{a}$, we derive the following orthogonality relation:

$$
h(\ell)=0 \quad \forall \ell \in \mathcal{L}, \quad p(r):=q_{1}(r)-q_{2}(r) .
$$

Step 2. We prove that the function $h_{1}(\ell)$, defined below, in formula $\left(3.65^{\prime}\right)$, is holomorphic in $\Pi_{+}:=$ $\{\ell: \ell \in \mathbb{C}, \Re \ell>0\}$ and belongs to class $N$ defined in (1.6).

Condition (1.28) implies $h_{1}(\ell)=0 \quad \forall \ell \in \mathcal{L}$. This implies by Theorem 1.3 that $h_{1}(\ell)=0 \quad \forall \ell \in \Pi_{+}$. Therefore, condition (1.28) implies $h(\ell)=0, \quad \ell=0,1,2, \ldots$, that is $(1.27)$ holds.

This implies, as we have proved above, that

$$
0=\int_{B_{a}} p(r) \psi_{1}(x, \alpha) \overline{\psi_{2}(x, \beta)} d x \quad \forall \alpha, \beta \in S^{2} .
$$

Equation (1.29) and property $C$ for the pair $\left\{L_{1}, L_{2}\right\}$ of the Schrödinger operators $L_{j}=-\nabla^{2}+q_{j}(x)-$ $1, \quad q_{j}(x) \in Q_{a}$, imply $p(r)=0$, that is, $q_{1}(r)=q_{2}(r)$.

An essential ingredient of our proof of the implication

$$
\left\{h_{1}(\ell)=0 \quad \forall \ell \in \mathcal{L}\right\} \Rightarrow\left\{h_{1}(\ell)=0 \quad \forall \ell=0,1,2,3, \ldots .\right\} .
$$

The proof of this implication is based on the existence of the transformation operators whose kernel does not depend on $\ell$. Existence and uniqueness of such operators as well as the estimate (3.53) (see section 3 below), which we use in the proof of the above implication, are established in section 3 . These results, although new and of independent interest, play an auxiliary role in our proof. They are presented in sections 3.3 and 3.4 as a part of the proof.

The description of the idea of our proof is complete.

In section 3 we derive the orthogonality relation (1.28).

In the same section we study the analytic properties of $h(\ell)$ as a function of complex $\ell$. In this study there are two basic steps.

First, we study the function

$$
h_{0}(\ell):=\int_{0}^{a} d r p(r) u_{\ell}^{2}(r), \quad u_{\ell}(r):=\sqrt{\frac{\pi r}{2}} J_{\ell+\frac{1}{2}}(r),
$$

where $J_{\ell+\frac{1}{2}}(r)$ is the standard Bessel function.

Note that $\varphi_{\ell}(r)=u_{\ell}(r)$ if $q(r)=0$.

Define

$$
H(\ell):=h_{0}(\ell)\left[\sqrt{\frac{2}{\pi}} \Gamma\left(\frac{1}{2}\right) 2^{\ell+\frac{1}{2}} \Gamma(\ell+1)\right]^{2},
$$

where $\Gamma(z)$ is the Gamma-function.

We prove:

Lemma 1.1. The function $H(\ell)$ is holomorphic in $\Pi_{+}$and $H(\ell) \in N$

Secondly, we prove the existence of the transformation operator, which sends $u_{\ell}(r)$ into $\varphi_{\ell}(r)$ :

$$
\varphi_{\ell}(r)=u_{\ell}(r)+\int_{0}^{r} K(r, \rho) u_{\ell}(\rho) \rho^{-2} d \rho, \quad K(r, 0)=0 .
$$

It is crucial for our argument that $K(r, \rho)$ does not depend on $\ell$. Therefore, the analytic properties of $h_{1}(\ell):=h(\ell)\left[\Gamma\left(\frac{1}{2}\right) \Gamma\left(\ell+\frac{1}{2}\right) 2^{\ell+\frac{1}{2}} \sqrt{\frac{2}{\pi}}\right]^{2}$ and $H(\ell)$, as functions of $\ell$, are essentially the same: these two functions are both holomorphic in $\Pi_{+}$and belong to class $N$.

In section 3 we prove some technical estimates for the kernel $K(r, \rho)$ of the transformation operator.

Although the transformation operators of the type (1.32) appeared formally earlier in the physical literature [CS, p.185], their existence was not proved. In the literature there exists a construction of the transformation operators whose kernels depend on $\ell$, see $[\mathrm{V}],[\mathrm{Le}],[\mathrm{M}]$. 
The difficulty of the existence proof for the transformation operator, whose kernel $K(r, \rho)$ does not depend on $\ell$, comes from the fact that the Goursat-type problem which one can derive for $K(r, \rho)$ involves differential operators with variable coefficients which degenerate at the origin.

We overcome this difficulty by introducing new variables and reducing the problem to an equivalent Volterra-type integral equation.

Existence and uniqueness of the solution to this equation are established in section 3, where some estimates of the solution are given.

This concludes the introduction.

In section 2 we state the basic uniqueness result.

In section 3 proofs are given.

\section{Statement of the Basic Result}

Let us assume that

$$
q(x) \in Q:=\left\{q: q \in Q_{a}, \quad q(x)=q(r), r:=|x|\right\},
$$

and let $\delta_{\ell}$ denote the fixed-energy phase shifts. Note that if $q \in Q$, then $\int_{0}^{a} r|q(r)| d r<c<\infty$. Here and below $c>0$ stands for various estimation constants.

The inverse scattering problem we are interested in can now be formulated:

ISP. Given the data $\left\{\delta_{\ell}\right\}_{\forall \ell \in \mathcal{L}}$, where $\mathcal{L}$ satisfies condition (1.1), can one recover $q(r) \in Q$ uniquely?

Our basic result is:

Theorem 2.1. Let $\mathcal{L}$ be an arbitrary fixed subset of non-negative integers which satisfies condition (1.1). Then the data $\left\{\delta_{\ell}\right\}_{\forall \ell \in \mathcal{L}}$, corresponding to a $q(r) \in Q$, determine $q(r)$ uniquely.

This result implies, in particular, that there is no $q(r) \in Q, \quad q(r) \not \equiv 0$, such that $\delta_{2 \ell}=0 \quad \forall \ell=$ $0,1,2, \ldots$

It also implies that there is no $q(r) \in Q$, such that $\delta_{0} \neq 0, \delta_{\ell}=0, \ell=1,2,3, \ldots$, which means that there are no potentials in $Q$ producing the scattering amplitude $A\left(\alpha, \alpha^{\prime}\right)$ which is constant for all $\alpha, \alpha^{\prime} \in S^{2}$ at a fixed positive energy, see also [R6] where this was proved for the first time by a different argument.

\section{Proofs}

\subsection{Proof of the orthogonality relation (1.28).}

Suppose $q_{1}(r)$ and $q_{2}(r)$ generate the same data $\left\{\delta_{\ell}\right\}_{\forall \ell \in \mathcal{L}}$. Subtract from equation (1.17) with $q=q_{1}$ and $\psi_{\ell}=\psi_{1 \ell}$ similar equation with $q=q_{2}$ and $\psi_{\ell}=\psi_{2 \ell}$ to get:

$$
\psi_{\ell}^{\prime \prime}+\psi_{\ell}-\frac{\ell(\ell+1)}{r^{2}} \psi_{\ell}-q_{1} \psi_{\ell}=p(r) \psi_{2 \ell},
$$

where

$$
\psi_{\ell}:=\psi_{1 \ell}-\psi_{2 \ell}, \quad p(r):=q_{1}(r)-q_{2}(r) .
$$

Multiply $(3.1)$ by $\psi_{1 \ell}(r)$, integrate over $(0, \infty)$ and then by parts using (1.18) and (1.19) and the assumption that $\delta_{\ell}$ is the same for $\psi_{1 \ell}$ and $\psi_{2 \ell}$ for $\ell \in \mathcal{L}$. The result is

$$
0=\int_{0}^{a} p(r) \psi_{1 \ell}(r) \psi_{2 \ell}(r) d r \quad \forall \ell \in \mathcal{L} .
$$

This is equivalent to the desired relation (1.28) because of (1.26).

\subsection{Analytic properties of the function $H(\ell)$.}

Proof of Lemma 1.1. Recall the well-known formula [GR, 8.411.8]:

$$
\sqrt{\frac{2}{\pi}} \Gamma\left(\frac{1}{2}\right) 2^{\ell+\frac{1}{2}} \Gamma(\ell+1) u_{\ell}(r)=r^{\ell+1} \int_{-1}^{1}\left(1-t^{2}\right)^{\ell} e^{i r t} d t .
$$


From (1.30), (1.31), and (3.4) one gets:

$$
H(\ell)=\int_{0}^{a} d r p(r) r^{2 \ell+2}\left(\int_{-1}^{1}\left(1-t^{2}\right)^{\ell} e^{i r t} d t\right)^{2} .
$$

Let $\ell=\sigma+i \tau, \quad \sigma \geq 0$. Then $H(\ell)$ is a holomorphic function of $\ell$ for $\sigma>0$ and

$$
|H(\ell)| \leq \int_{0}^{a} d r|p(r)| r a^{2 \sigma+1} \leq c a^{2 \sigma} .
$$

One can always assume $a>1$ since $\sigma>0$.

Let us check that (3.6) implies that $H(\ell) \in N$. One has

$$
\ln ^{+}(a b) \leq \ln ^{+} a+\ln ^{+} b \quad \text { for } a, b>0 .
$$

Therefore, using (3.6), one obtains:

$$
\begin{aligned}
& \int_{-\pi}^{\pi} \ln ^{+}\left|H\left(\frac{1-r e^{i \varphi}}{1+r e^{i \varphi}}\right)\right| d \varphi \leq \int_{-\pi}^{\pi} \ln ^{+}\left|c a^{2 R e \frac{1-r e^{i \varphi}}{1+r e^{i \varphi}}}\right| d \varphi \leq \\
& \leq c_{1}+2 \ln a \int_{-\pi}^{\pi} \operatorname{Re} \frac{1-r e^{i \varphi}}{1+r e^{i \varphi}} d \varphi \leq c_{1}+2 \ln a \int_{-\pi}^{\pi} \frac{1-r^{2}}{1+r^{2}+2 r \cos \varphi} d \varphi=c_{1}+4 \pi \ln a<\infty, a>1 .
\end{aligned}
$$

Here we have used the known formula

$$
\int_{-\pi}^{\pi} \frac{d \varphi}{1+r^{2}+2 r \cos \varphi}=\frac{2 \pi}{1-r^{2}}, \quad 0<r<1,
$$

which is easy to check.

Estimate (3.7) proves that $H(\ell) \in N$. Lemma 1.1 is proved.

\subsection{Transformation operators.}

Define

$$
L_{r} \varphi:=\left[r^{2} \frac{\partial^{2}}{\partial r^{2}}+r^{2}-r^{2} q(r)\right] \varphi:=L_{0 r} \varphi-r^{2} q(r) \varphi .
$$

For the regular solution to (1.17) one has the following differential equation:

$$
L_{r} \varphi_{\ell}(r)=\ell(\ell+1) \varphi_{\ell}(r) \text {, }
$$

and for the function $u_{\ell}(r)=\sqrt{\frac{\pi r}{2}} J_{\ell+\frac{1}{2}}(r)$ the equation

$$
L_{0 r} u_{\ell}(r)=\ell(\ell+1) u_{\ell}(r) .
$$

Let us look for the kernel $K(r, \rho)$ such that formula (1.32) gives the regular solution to equation (1.17). Substitute (1.32) into (1.17), drop index $\ell$ for convenience, use (3.10) and (3.11), and get

$$
\begin{aligned}
0=-r^{2} q(r) u+\left(r^{2}-r^{2} q(r)\right) \int_{0}^{r} K(r, \rho) u \rho^{-2} d \rho & \\
& -\int_{0}^{r} K(r, \rho) \rho^{-2} L_{0 \rho} u d \rho+r^{2} \partial_{r}^{2} \int_{0}^{r} K(r, \rho) u \rho^{-2} d \rho .
\end{aligned}
$$

We assume first that $K(r, \rho)$ is twice continuously differentiable with respect to its variables in the region $0<r<\infty, \quad 0<\rho \leq r$. This assumption requires extra smoothness of $q(r), \quad q(r) \in C^{1}(0, a)$. If $q(r)$ satisfies condition (2.1), then equation (3.18) below has to be understood in the sense of distributions. Eventually we will work with an integral equation (3.45) (see below) for which assumption (2.1) suffices. 
Note that

$$
\int_{0}^{r} K(r, \rho) \rho^{-2} L_{0 \rho} u d \rho=\int_{0}^{r} L_{0 \rho} K(r, \rho) u \rho^{-2} d \rho+K(r, r) u_{r}-K_{\rho}(r, r) u
$$

provided that

$$
K(r, 0)=0
$$

We assume (3.14) to be valid.

Denote

$$
\dot{K}:=\frac{d K(r, r)}{d r}
$$

Then

$$
\begin{aligned}
r^{2} \partial_{r}^{2} \int_{0}^{r} K(r, \rho) u \rho^{-2} d \rho=\dot{K} u+K(r, r) u_{r}-\frac{2}{r} K(r, r) u+ & \\
& K_{r}(r, r) u+r^{2} \int_{0}^{r} K_{r r}(r, \rho) u \rho^{-2} d \rho .
\end{aligned}
$$

Combining (3.12)-(3.16) and writing again $u_{\ell}$ in place of $u$, one gets

$$
\begin{aligned}
0=\int_{0}^{r}\left[L_{r} K(r, \rho)-L_{0 \rho} K(r, \rho)\right] u_{\ell}(\rho) \rho^{-2} d \rho+u_{\ell}(r)\left[-r^{2} q(r)+\dot{K}-\right. & \\
& \left.\frac{2 K_{r}(r, r)}{r}+K_{r}(r, r)+K_{\rho}(r, r)\right], \quad \forall r>0, \quad \ell=0,1,2, \ldots .
\end{aligned}
$$

Let us prove that (3.17) implies:

$$
\begin{gathered}
L_{r} K(r, \rho)=L_{0 \rho} K(r, \rho), \quad 0<\rho \leq r, \\
q(r)=\frac{2 \dot{K}}{r^{2}}-\frac{2 K(r, r)}{r}=\frac{2}{r} \frac{d}{d r} \frac{K(r, r)}{r} .
\end{gathered}
$$

This proof requires a lemma.

Lemma 3.1. Assume that $\rho f(\rho) \in L^{1}(0, r)$ and $\rho A(\rho) \in L^{1}(0, r)$. If

$$
0=\int_{0}^{r} f(\rho) u_{\ell}(\rho) d \rho+u_{\ell}(r) A(r) \quad \forall \ell=0,1,2, \ldots
$$

then

$$
f(\rho) \equiv 0 \text { and } A(r)=0
$$

Proof. Equations (3.20) and (3.4) imply:

$$
\begin{gathered}
0=\int_{-1}^{1} d t\left(1-t^{2}\right)^{\ell}\left(\frac{d}{i d t}\right)^{\ell} \int_{0}^{r} d \rho \rho f(\rho) e^{i \rho t}+ \\
\quad r A(r) \int_{-1}^{1}\left(1-t^{2}\right)^{\ell}\left(\frac{d}{i d t}\right)^{\ell} e^{i r t} d t
\end{gathered}
$$

Therefore

$$
0=\int_{-1}^{1} d t \frac{d^{\ell}\left(t^{2}-1\right)^{\ell}}{d t^{\ell}}\left[\int_{0}^{r} d \rho \rho f(\rho) e^{i \rho t}+r A(r) e^{i r t}\right], \quad l=0,1,2, \ldots
$$

Recall that the Legendre polynomials are defined by the formula

$$
P_{\ell}(t)=\frac{1}{2^{\ell} !} \frac{d^{\ell}}{d t^{\ell}}\left(t^{2}-1\right)^{\ell}
$$


and they form a complete system in $L^{2}(-1,1)$.

Therefore (3.22) implies

$$
\int_{0}^{r} d \rho \rho f(\rho) e^{i \rho t}+r A(r) e^{i r t}=0 \quad \forall t \in[-1,1] .
$$

Equation (3.24)implies

$$
\int_{0}^{r} d \rho \rho f(\rho) e^{i \rho t}=0, \quad \forall t \in[-1,1]
$$

and

$$
r A(r)=0 .
$$

Therefore $A(r)=0$. Also $f(\rho)=0$ because the left-hand side of $(3,25)$ is an entire function of $t$, which vanishes on the interval $[-1,1]$ and, consequently, it vanishes identically, so that $\rho f(\rho)=0$ and therefore $f(\rho) \equiv 0$.

Lemma 3.1 is proved

\subsection{Existence and uniqueness of the transformation operators.}

Let us prove that the problem (3.18), (3.19), (3.14), which is a Goursat-type problem, has a solution and this solution is unique in the class of functions $K(r, \rho)$, which are twice continuously differentiable with respect to $\rho$ and $r, \quad 0<r<\infty, \quad 0<\rho \leq r$. In this section we assume that $q(r) \in C^{1}(0, a)$. This assumption implies that $K(r, \rho)$ is twice continuously differentialable. If $q(r) \in Q$, see (2.1), the arguments in this section which deal with integral equation (3.45) remain valid. Specifically, existence and uniqueness of the solution to equation (3.45) is proved under the only assumption $\int_{0}^{a} r|q(r)| d r<\infty$ as far as the smoothness of $q(r)$ is concerned.

By a limiting argument one can reduce the smoothness requirements on $q$ to the condition (2.1) but in this case equation (3.18) has to be understood in distributional sense.

Let us rewrite the problem we want to study:

$$
\begin{gathered}
r^{2} K_{r r}-\rho^{2} K_{\rho \rho}+\left[r^{2}-r^{2} q(r)-\rho^{2}\right] K(r, \rho)=0, \quad 0<\rho \leq r, \\
K(r, r)=\frac{r}{2} \int_{0}^{r} s q(s) d s:=g(r), \\
K(r, 0)=0 .
\end{gathered}
$$

The difficulty in the study of this Goursat-type problem comes from the fact that the coefficients in front of the second derivatives of the kernel $K(r, \rho)$ are variable.

Let us reduce problem (3.27)-(3.29) to the one with constant coefficients. To do this, introduce the new variables:

$$
\xi=\ln r+\ln \rho, \quad \eta=\ln r-\ln \rho .
$$

Note that

$$
\begin{gathered}
r=e^{\frac{\xi+\eta}{2}}, \quad \rho=e^{\frac{\xi-\eta}{2}}, \\
\eta \geq 0, \quad-\infty<\xi<\infty,
\end{gathered}
$$

and

$$
\partial_{r}=\frac{1}{r}\left(\partial_{\xi}+\partial_{\eta}\right), \quad \partial_{\rho}=\frac{1}{\rho}\left(\partial_{\xi}-\partial_{\eta}\right) .
$$

Let

$$
K(r, \rho):=B(\xi, \eta) .
$$

A routine calculation transforms equations (3.27)-(3.29) to the following ones:

$$
B_{\xi \eta}(\xi, \eta)-\frac{1}{2} B_{\eta}(\xi, \eta)+Q(\xi, \eta) B=0, \quad \eta \geq 0, \quad-\infty<\xi<\infty,
$$




$$
\begin{gathered}
B(\xi, 0)=g\left(e^{\frac{\xi}{2}}\right):=G(\xi), \quad-\infty<\xi<\infty \\
B(-\infty, \eta)=0, \quad \eta \geq 0,
\end{gathered}
$$

where $g(r)$ is defined in (3.28).

Here we have defined

$$
Q(\xi, \eta):=\frac{1}{4}\left[e^{\xi+\eta}-e^{\xi+\eta} q\left(e^{\frac{\xi+\eta}{2}}\right)-e^{\xi-\eta}\right],
$$

and took into account that $\rho=r$ implies $\eta=0$, while $\rho=0$ implies, for any fixed $\eta \geq 0$, that $\xi=-\infty$.

Note that

$$
\begin{gathered}
\sup _{-\infty<\xi<\infty} e^{-\frac{\xi}{2}} G(\xi)<c, \\
\sup _{0 \leq \eta \leq B} \int_{-\infty}^{A}|Q(s, \eta)| d s \leq c(A, B),
\end{gathered}
$$

for any $A \in \mathbb{R}$ and $B>0$, where $c(A, B)>0$ is a constant.

To get rid of the second term on the left-hand side of (3.34), let us introduce the new kernel $L(\xi, \eta)$ by the formula:

$$
L(\xi, \eta):=B(\xi, \eta) e^{-\frac{\xi}{2}} .
$$

Then (3.34)-(3.36) can be written as:

$$
\begin{gathered}
L_{\eta \xi}(\xi, \eta)+Q(\xi, \eta) L(\xi, \eta)=0, \quad \eta \geq 0, \quad-\infty<\xi<\infty, \\
L(\xi, 0)=e^{-\frac{\xi}{2}} G(\xi):=b(\xi):=\frac{1}{2} \int_{0}^{e^{\frac{\xi}{2}}} s q(s) d s, \quad-\infty<\xi<\infty, \\
L(-\infty, \eta)=0, \quad \eta \geq 0 .
\end{gathered}
$$

We want to prove existence and uniqueness of the solution to (3.41)-(3.43). In order to choose a convenient Banach space in which to work, let us transform problem (3.41)-(3.43) to an equivalent Volterratype integral equation.

Integrate (3.41) with respect to $\eta$ from 0 to $\eta$ and use (3.42) to get

$$
L_{\xi}(\xi, \eta)-b^{\prime}(\xi)+\int_{0}^{\eta} Q(\xi, t) L(\xi, t) d t=0 .
$$

Integrate (3.44) with respect to $\xi$ from $-\infty$ to $\xi$ and use (3.44) to get

$$
L(\xi, \eta)=-\int_{-\infty}^{\xi} d s \int_{0}^{\eta} d t Q(s, t) L(s, t)+b(\xi):=V L+b,
$$

where

$$
V L:=-\int_{-\infty}^{\xi} d s \int_{0}^{\eta} d t Q(s, t) L(s, t) .
$$

Consider the space $X$ of continuous functions $L(\xi, \eta)$, defined in the half-plane $\eta \geq 0, \quad-\infty<\xi<\infty$, such that for any $B>0$ and any $-\infty<A<\infty$ one has

$$
\|L\|:=\|L\|_{A B}:=\sup _{\substack{0 \leq t \leq B \\-\infty<s \leq A}}\left(e^{-\gamma t}|L(s, t)|\right)<\infty,
$$

where $\gamma>0$ is a number which will be chosen later so that that the operator $V$ in (3.45) will be a contraction mapping on the Banach space of functions with norm (3.47) for a fixed pair $A, B$. To choose $\gamma>0$, let us estimate the norm of $V$. One has:

$$
\begin{aligned}
\|V L\| \leq \sup _{-\infty<\xi \leq A, 0 \leq \eta \leq B} & \left(\int_{-\infty}^{\xi} d s \int_{0}^{\eta} d t|Q(s, t)| e^{-\gamma(\eta-t)} e^{-\gamma t}|L(s, t)|\right) \leq \\
& \leq\|L\| \sup _{-\infty<\xi \leq A, 0 \leq \eta \leq B} \int_{-\infty}^{\xi} d s \int_{0}^{\eta} d t\left(2 e^{s+t}+e^{s+t}\left|q\left(e^{\frac{s+t}{2}}\right)\right|\right) e^{-\gamma(\eta-t)} \leq \frac{c}{\gamma}\|L\|,
\end{aligned}
$$


where $c>0$ is a constant depending on $A, B$ and $\int_{0}^{a} r|q(r)| d r$. Indeed, one has:

$$
2 \int_{-\infty}^{A} d s \int_{0}^{\eta} d t e^{s+t-\gamma(\eta-t)}=2 e^{A} \int_{0}^{\eta} d t e^{t-\gamma(\eta-t)} d t \leq 2 e^{A+B} \frac{1-e^{-\gamma B}}{\gamma}=\frac{c_{1}}{\gamma},
$$

and, using the substitution $\sigma=e^{\frac{s+t}{2}}$, one gets:

$$
\begin{gathered}
\int_{-\infty}^{A} d s \int_{0}^{\eta} d t e^{s+t}\left|q\left(e^{\frac{s+t}{2}}\right)\right| e^{-\gamma(\eta-t)}= \\
=\int_{0}^{\eta} d t e^{-\gamma(\eta-t)} \int_{-\infty}^{A} d s e^{s+t}\left|q\left(e^{\frac{s+t}{2}}\right)\right|= \\
=2 \int_{0}^{\eta} d t e^{-\gamma(\eta-t)} \int_{0}^{e^{\frac{A+t}{2}}} d \sigma \sigma|q(\sigma)|= \\
\quad=\frac{2\left(1-e^{-\gamma B}\right)}{\gamma} \int_{0}^{a} d \sigma \sigma|q(\sigma)|:=\frac{c_{2}}{\gamma} .
\end{gathered}
$$

From these estimates inequality (3.48) follows.

It follows from (3.48) that $V$ is a contraction mapping in the space $X_{A B}$ of continuous functions in the region $-\infty<\xi \leq A, \quad 0 \leq \eta \leq B$, with the norm (3.47) provided that

$$
\gamma>c .
$$

Therefore equation (3.45) has a unique solution $L(\xi, \eta)$ in the region

$$
-\infty<\xi<A, \quad 0 \leq \eta \leq B
$$

for any real $A$ and $B>0$ if (3.50) holds. This means that the above solution is defined for any $\xi \in \mathbb{R}$ and any $\eta \geq 0$.

Equation (3.45) is equivalent to problem (3.41)-(3.43) and, by (3.40), one has:

$$
B(\xi, \eta)=L(\xi, \eta) e^{\frac{\xi}{2}}
$$

Therefore we have proved the existence and uniqueness of $B(\xi, \eta)$, that is, of the kernel $K(r, \rho)=B(\xi, \eta)$ of the transformation operator (1.32). Recall that $r$ and $\rho$ are related to $\xi$ and $\eta$ by formulas (3.31).

Let us formulate the result:

Lemma 3.2. The kernel of the transformation operator (1.32) solves problem (3.27)-(3.29). The solution to this problem does exist and is unique for any potential $q(r) \in C^{1}(0, a)$ in the class of twice continuously differentiable functions. If $q(r) \in L^{\infty}(0, a)$, then $K(r, \rho)$ has first derivatives which are bounded and equation (3.27) has to be understood in the sense of distributions. The following estimate holds for any $r>0$ :

$$
\int_{0}^{r}|K(r, \rho)| \rho^{-1} d \rho<\infty .
$$

Proof of Lemma 3.2. We have already proved all the assertions of Lemma 3.2 except estimate (3.53). Let us prove this estimate.

Note that

$$
\int_{0}^{r}|K(r, \rho)| \rho^{-1} d \rho=r \int_{0}^{\infty}|L(2 \ln r-\eta, \eta)| e^{-\frac{\eta}{2}} d \eta<\infty
$$

Indeed, if $r>0$ is fixed, then, by (3.31), $\xi+\eta=2 \ln r=$ const. Therefore $d \xi=-d \eta$, and $\rho^{-1} d \rho=$ $\frac{1}{2}(d \xi-d \eta)=-d \eta, \quad \xi=2 \ln r-\eta$. Thus:

$$
\int_{0}^{r}|K(r, \rho)| \rho^{-1} d \rho=\int_{0}^{\infty}|L(2 \ln r-\eta, \eta)| e^{\frac{2 \ln r-\eta}{2}} d \eta=r \int_{0}^{\infty}|L(2 \ln r-\eta, \eta)| e^{-\frac{\eta}{2}} d \eta .
$$

The following estimate holds:

$$
|L(\xi, \eta)| \leq c e^{\left(2+\epsilon_{1}\right)\left[\eta \mu_{1}(\xi+\eta)\right]^{\frac{1}{2}+\epsilon_{2}}},
$$

where $\epsilon_{j}>0, j=1,2$, are arbitrarily small numbers and $\mu_{1}$ is defined in formula (3.60) below, see also formula (3.58) for the definition of $\mu$.

Estimate (3.56) is proved below, in Lemma 3.3.

From (3.55) and (3.56) estimate (3.53) follows. Lemma 3.2 is proved. 
Lemma 3.3. Estimate (3.56) holds.

Proof of Lemma 3.3. From (3.45) one gets:

$$
m(\xi, \eta) \leq c_{0}+(W m)(\xi, \eta), \quad m(\xi, \eta):=|L(\xi, \eta)|,
$$

where $c_{0}=\sup _{-\infty<\xi<\infty}|b(\xi)| \leq \frac{1}{2} \int_{0}^{a} s|q(s)| d s$ (see (3.42)), and

$$
W m:=\int_{-\infty}^{\xi} d s \int_{0}^{\eta} d t \mu(s+t) m(s, t), \quad \mu(s):=\frac{1}{2} e^{s}\left(1+\left|q\left(e^{\frac{s}{2}}\right)\right|\right) .
$$

It is sufficient to consider inequality (3.57) with $c_{0}=1$ : if $c_{0}=1$ and the solution $m_{0}(\xi, \eta)$ to (3.57) satisfies (3.56) with $c=c_{1}$, then the solution $m(\xi, \eta)$ of (3.57) with any $c_{0}>0$ satisfies (3.56) with $c=c_{0} c_{1}$.

Therefore, assume that $c_{0}=1$, then (3.57) reduces to:

$$
m(\xi, \eta) \leq 1+(W m)(\xi, \eta)
$$

Inequality (3.56) follows from (3.59) by iterations. Let us give the details.

Note that

$$
W 1=\int_{-\infty}^{\xi} d s \int_{0}^{\eta} d t \mu(s+t)=\int_{0}^{\eta} d t \int_{-\infty}^{\xi} d s \mu(s+t)=\int_{0}^{\eta} d t \mu_{1}(\xi+t) \leq \eta \mu_{1}(\xi+\eta) .
$$

Here we have used the notation

$$
\mu_{1}(\xi)=\int_{-\infty}^{\xi} \mu(s) d s
$$

and the fact that $\mu_{1}(s)$ is a monotonically increasing function, since $\mu(s)>0$. Note also that $\mu_{1}(s)<\infty$ for any $s,-\infty<s<\infty$.

Furthermore,

$$
W^{2} 1 \leq \int_{-\infty}^{\xi} d s \int_{0}^{\eta} d t \mu(s+t) t \mu_{1}(s+t) \leq \int_{0}^{\eta} d t t \int_{-\infty}^{\xi} d s \mu(s+t) \mu_{1}(s+t)=\frac{\eta^{2}}{2 !} \frac{\mu_{1}^{2}(\xi+\eta)}{2 !} .
$$

Let us prove by induction that

$$
W^{n} 1 \leq \frac{\eta^{n}}{n !} \frac{\mu_{1}^{n}(\xi+\eta)}{n !} .
$$

For $n=1$ and $n=2$ we have checked (3.62). Suppose (3.62) holds for some $n$, then

$$
W^{n+1} 1 \leq W\left(\frac{\eta^{n}}{n !} \frac{\mu_{1}^{n}(\xi+\eta)}{n !}\right)=\int_{0}^{\eta} d t \frac{t^{n}}{n !} \int_{-\infty}^{\xi} d s \mu(s+t) \frac{\mu_{1}^{n}(s+t)}{n !} \leq \frac{\eta^{n+1}}{(n+1) !} \frac{\mu_{1}^{n+1}(\xi+\eta)}{(n+1) !} .
$$

By induction, estimate (3.61) is proved for all $n=1,2,3, \ldots$. Therefore (3.59) implies

$$
m(\xi, \eta) \leq 1+\sum_{n=1}^{\infty} \frac{\eta^{n}}{n !} \frac{\mu_{1}^{n}(\xi+\eta)}{n !} \leq c e^{\left(2+\epsilon_{1}\right)\left[\eta \mu_{1}(\eta+\xi)\right]^{\frac{1}{2}+\epsilon_{2}}}
$$

where we have used Theorem 2 from [L, section 1.2], namely the order of the entire function $F(z):=$ $1+\sum_{n=1}^{\infty} \frac{z^{n}}{(n !)^{2}}$ is $\frac{1}{2}$ and its type is 2 . The constant $c>0$ in (3.56) depends on $\epsilon_{j}, j=1,2$.

Recall that the order of an entire function $F(z)$ is the number $\rho:=\limsup _{r \rightarrow \infty} \frac{\ln \ln M_{F}(r)}{\ln r}$, where $M_{F}(r):=\max _{|z|=r}|F(z)|$. The type of $F(z)$ is the number $\sigma:=\limsup _{r \rightarrow \infty} \frac{\ln M_{F}(r)}{r^{\rho}}$. It is known [L], that if $F(z)=\sum_{n=0}^{\infty} c_{n} z^{n}$ is an entire function, then its order $\rho$ and type $\sigma$ can be calculated by the formulas:

$$
\rho=\limsup _{n \rightarrow \infty} \frac{n \ln n}{\ln \frac{1}{\left|c_{n}\right|}}, \quad \sigma=\frac{\limsup _{n \rightarrow \infty}\left(n\left|c_{n}\right|^{\frac{\rho}{n}}\right)}{e \rho} .
$$

If $c_{n}=\frac{1}{(n !)^{2}}$, then the above formulas yield $\rho=\frac{1}{2}$ and $\sigma=2$. Lemma 3.3 is proved. 


\subsection{Proof of Theorem 2.1.}

Suppose that there are two potentials which generate the same data $\left\{\delta_{\ell}\right\}_{\forall \ell \in \mathcal{L}}$. In section 3.1 we have proved that this implies (3.3). From (3.3) and (1.26) it follows that (3.3) is equivalent to (1.28).

From Lemma 3.2, formula (1.32), Lemma 1.1, and the definition

$$
h(\ell)=\int_{0}^{a} d r p(r) \varphi_{1 \ell}(r) \varphi_{2 \ell}(r),
$$

it follows that the function

$$
h_{1}(\ell):=\left[\sqrt{\frac{2}{\pi}} \Gamma\left(\frac{1}{2}\right) \Gamma(\ell+1) 2^{\ell+\frac{1}{2}}\right]^{2} h(\ell) \in N .
$$

This is checked as in the proof of Lemma 1.1 in section 3.2. There are four terms which one gets from multiplication of $\varphi_{1 \ell}(r)$ by $\varphi_{2 \ell}(r)$, where $\varphi_{j \ell}(r), j=1,2$, are expressed by formula (1.32) with $K(r, \rho)=K_{j}(r, \rho), j=1,2$. The first term contains $u_{\ell}^{2}(r)$ and is identical with (1.30), the second and third terms contain the products of the type $u_{\ell}(r) u_{\ell}(\rho)$, while the fourth term contains the term $u_{\ell}\left(\rho_{1}\right) u_{\ell}\left(\rho_{2}\right)$. These terms are treated like in the proof of Lemma 1.1. and estimate (3.53) is used.

From (3.65'), Theorem 1.3, and assumption (1.28) it follows that $h(\ell)=0$ for $\ell=0,1,2,3, \ldots$.

From this and (1.26) it follows that (1.23) holds for $\ell=0,1,2,3, \ldots$

From (1.23) and (1.22) it follows that

$$
\int_{B_{a}} d x p(r) \psi_{1}(x, \alpha) \overline{\psi_{2}(x, \beta)}=0 \quad \forall \alpha, \beta \in S^{2} .
$$

From (3.66) and Theorem 1.2 one concludes that $p(r)=0$.

Theorem 2.1 is proved.

\subsection{Heuristic motivation of the basic result.}

Here we give an heuristic motivation of the basic result, namely of Theorem 2.1.

It is well known that

$$
u_{\ell}(r):=\sqrt{\frac{\pi r}{2}} J_{\ell+\frac{1}{2}}(r)=\sqrt{\frac{r}{2}}\left(\frac{e r}{2 \ell+1}\right)^{\frac{2 \ell+1}{2}} \frac{1}{\sqrt{2 \ell+1}}[1+o(1)] \text { as } \ell \rightarrow \infty .
$$

One can prove that $\varphi_{\ell}(r)$ has the same asymptotics

$$
\varphi_{\ell}(r)=\sqrt{\frac{r}{2}}\left(\frac{e r}{2 \ell+1}\right)^{\frac{2 \ell+1}{2}} \frac{1}{\sqrt{2 \ell+1}}[1+o(1)] \text { as } \ell \rightarrow \infty .
$$

If one substitutes (3.68) into (1.28), one gets

$$
0=\int_{0}^{a} d r r^{2} p(r) r^{2 \ell}[1+o(1)] \quad \forall \ell \in \mathcal{L} .
$$

If one neglects the term $o(1)$, then one gets

$$
0=\int_{0}^{a} d r r^{2} p(r) r^{2 \ell} \quad \forall \ell \in \mathcal{L}
$$

From (3.70) and the well known Müntz's theorem [Ru, p.336], it follows that $p(r)=0$, which yields the conclusion of Theorem 2.1.

This heuristic argument is not a proof because the justification of the passage from (3.69) to (3.70) is not given and is not clear if such a justification can be given directly. Our proof of Theorem 2.1 can be considered as an indirect justification of this heuristic argument.

It is known that condition (1.1) is necessary and sufficient for completeness of the set $\left\{r^{\ell}\right\}_{\ell \in \mathcal{L}}$ in $L_{1}(0, a)$ for any fixed $a>0$.

Therefore one can raise the following question:

Is it true that condition (1.1) is necessary for the conclusion of Theorem 2.1 to be valid?

This interesting question is open. 


\section{REFERENCES}

[A] Airapetyan R., Ramm A.G. and Smirnova A.B., Example of two different potentials which have practical the same fixed-energy phase shifts, Phys. Lett A 254 (1999), no. 3-4, 141-148.

[CS]Chadan K., Sabatier P., Inverse problems in Quantum scattering theory, Springer Verlag, New York, 1989.

[GRGGradshteyn I., Ryzhik I., Table of integrals, series and products, Acad. Press, Boston, 1994.

[L] Levin B., Distribution of zeros of entire functions, AMS Transl. vol. 5, Providence RI, 1980.

[Le] Levitan B., Inverse Sturm-Liouville problems, VNU Press, Utrecht, The Netherlands, 1987.

[M] Marchenko V., Sturm-Liouville operators and applications, Birkhäser Verlag, Boston, 1986.

[R1]Ramm A.G., Recovery of the potential from fixed energy scattering data, Inverse Problems 4 (1988), 877-886.

[R2]Ramm A.G., Multidimensional inverse scattering problems, Longman/Wiley, New York, 1992, pp. 1-385; Multidimensional inverse scattering problems, Mir Publishers, Moscow, 1994, pp. 1-496 (Russian translation of the expanded monograph).

[R3]Ramm A.G., Completeness of the products of solutions of PDE and inverse problems, Inverse Problems 6 (1990), 643-664.

[R4]Ramm A.G., Necessary and sufficient condition for a PDE to have property C, J. Math. Anal. Appl. 156 (1991), 505-509.

[R5]Ramm A.G., Stability estimates in inverse scattering, Acta Appl. Math. 28 (1992), no. 1, 1-42.

[R6]Ramm A.G., Can a constant be a scattering amplitude?, Phys. Lett. 154A (1991), 35-37.

[Ru]Rudin W., Real and complex analysis, McGraw Hill, New York, 1974.

[V] Volk V., On inverse formulas for a differential equation with a singularity at $x=0$, Uspekhi Math. Nauk 8 (1953), no. 4, 1141-151 (in Russian). 